\title{
Real-World Evidence Studies of Oritavancin Use in Gram-Positive Infections Augment Randomized Controlled Trials to Address Clinical and Economic Outcomes
}

\author{
Mark Redell ${ }^{1}$
}

Published online: 25 June 2020

(c) The Author(s) 2020

\begin{abstract}
Randomized controlled trials (RCTs) sponsored by pharmaceutical manufacturers for regulatory approval are conducted with restrictive criteria in an effort to definitively demonstrate the safety and efficacy of a drug or biologic. Unfortunately, the strict enrollment criteria in RCTs may exclude patients likely to receive the medication in a real-world clinical practice. Antibiotic RCTs for registration are designed to show noninferiority against standard of care or best available therapy, often minimizing clinical differentiation needed by clinicians to select the optimal agent for their patients. Lastly, RCTs do not include pharmacoeconomic data that would add a cost basis for determining the value of one product over another. Realworld studies may add support to the safety and efficacy demonstrated from RCTs and address patient populations excluded from clinical development programs. This supplement presents several real-world studies demonstrating the clinical and economic outcomes of various uses of oritavancin to augment the evidence published from RCTs. Clinicians may decide how to use this information in their own practice settings.
\end{abstract}

The randomized controlled trial (RCT) is a well-established method for gathering robust evidence of the safety and efficacy of medical interventions. Investigators study narrowly defined patient populations to reduce bias and confounding by utilizing randomization, blinding to subject allocation, and strict patient eligibility criteria $[1,2]$. The inclusion criteria for patient enrollment into RCTs excludes a significant number of patients typically seen in real-world practice, such as patients who are elderly, exhibit multiple comorbidities, exhibit complicated infections, are taking concomitant medications, or come from certain social or demographic settings [1]. Real-world evidence (RWE) studies provide a bridge for validation or dispute between RCTs and clinical practice and thereby may fill current gaps in clinical knowledge. While RCTs provide evidence of efficacy, real-world studies demonstrate comparative effectiveness, safety, and economic performance in a naturalistic setting. Real-world

Digital features To view digital features for this article go to https ://doi.org/10.6084/m9.figshare.12221690.

Mark Redell

mredell@melinta.com

1 Melinta Therapeutics, 44 Whippany Road, Suite 280, Morristown, NJ 07960, USA data collected from multiple sources, such as registries, electronic health records, administrative claim databases, pharmacy records, and social media, can complement the evidence from RCTs and augment clinical knowledge on medications already approved for use [1-3].

The International Society for Pharmacoeconomics and Outcomes Research (ISPOR) defines real-world data as "data obtained outside the context of randomized controlled trials generated during routine clinical practice [in which] patients and their clinicians choose treatments on the basis of the patient's clinical characteristics and preferences" [3, 4]. The US FDA has acknowledged the importance of clinical evidence derived from a variety of data sources, such as those listed above, by accepting real-world safety data and considering the use of real-world efficacy data for registration updates [5]. Section 505F of the 2st Century Cures Act establishes a framework for FDA evaluation of RWE that may accompany an application for a new drug indication or submission of postapproval study requirements [1, $5,6]$. The full impact of the 21 st Century Cures Act is still years away, but there is a clear intent of regulators to encourage the use of real-world data to inform drug development programs. In the case of antibiotics, RWE may be used to document the natural history of disease progress to identify problematic geographies, identify predictors of treatment 
response, and inform the most beneficial study interventions [7]. Other benefits derived from RWE studies include (1) less time to produce meaningful data, enabling adaptation to clinical practice sooner; (2) the ability to capture additional information (i.e., patient experience/journey) that may impact outcomes and conclusions; (3) the opportunity to identify uncommon adverse events or covariates not previously collected; and (4) the availability of economic data that may impact the provision of value-based healthcare. RCTs are notoriously expensive and difficult to execute. The expense and time incurred by conducting pivotal clinical trials has been growing steadily for years. Recently, Moore et al. [8] estimated that a clinical trial providing a basis for FDA approval submitted in 2015 and 2016, which assessed clinical benefit against an active comparator or placebo, had a mean cost of \$US35.1 million (95\% confidence interval 25.4-44.8). This cost is a small portion against the estimated sponsor's average out-of-pocket cost per approved new compound of \$US1395 million (year 2013 values) [9]. Stergiopoulos et al. [10] calculated that the fully loaded (direct and indirect) cost for a phase III hospital-acquired bacterial pneumonia and ventilator-associated bacterial pneumonia study was \$US89.6 million (year 2014 values), exceeding that of oncology and endocrine trials by more than twofold.

Overall, combining data from RCTs and RWE studies would allow decision makers to make more evidence-based decisions regarding the use of medications, including antibiotics. Using the right antibiotic for the right patient at the right time is critical to effectively treat all infection types and prevent the development of antimicrobial resistance. A thorough discussion of study design that incorporates real-world evidence is provided by Lee et al. [11]. RWE evaluated in the context of treatment guidelines can provide information on how infection management practices evolve over time. For example, Kamath et al. [12] noted that guidelines are difficult to interpret, do not fit individual circumstances, and do not include other approaches that are based on evidence or good clinical judgment. The authors pointed to the value of real-world studies to provide evidence of clinical outcomes and patient preferences for new therapeutic strategies that may be incorporated into real-world practice.

The studies in this supplement add to the evidence supporting the safe and effective use of single and multiple doses of oritavancin $\left(\right.$ ORBACTIV $^{\circledR}$, Melinta Therapeutics) in the treatment of acute bacterial skin and skin structure infection (ABSSSI), complicated skin and soft tissue infections, osteomyelitis, and other disease entities caused by Gram-positive bacteria [13]. Oritavancin is indicated for the treatment of adult patients with ABSSSI caused by susceptible isolates of Gram-positive microorganisms, including methicillin-resistant Staphylococcus aureus. It is administered as a single dose of $1200 \mathrm{mg}$ parenterally over $3 \mathrm{~h}$ [14]. The individual postmarketing and real-world experiences with oritavancin included herein provide additional resources to evaluate its safety and efficacy in patient populations not studied in the registrational clinical development program. This supplementary information may guide treatment decisions by addressing clinical knowledge gaps about oritavancin.

Four studies (Estrada et al. [15], Whittaker et al. [16], Helton et al. [17], and Brownell et al. [18]) report observations from real-world clinical settings in terms of either avoidance of hospitalization or early discharge from an inpatient setting by use of single-dose oritavancin for treatment of ABSSSI. Oritavancin was administered in outpatient parenteral antimicrobial therapy programs, emergency departments (EDs), or last day hospital stays in a total of 451 patients with ABSSSI. All-cause or infection-related 30-day readmissions to the hospital or ED were also tracked. All four studies compared outcomes associated with oritavancin to either historical or parallel control groups to assess clinical and economic outcomes. Importantly, these studies were undertaken following identification of patients in their respective institutions who remained hospitalized solely for the purpose of parenteral antibiotic therapy despite clinical stability with significant infection resolution of ABSSSIs. The value of internal quality assurance review is a key characteristic of the study by Whittaker et al. [16] in devising programs that can lower healthcare costs. Overall, the ABSSSI-focused studies reveal methodologies that may be implemented by a variety of healthcare institutions to achieve early and directed patient discharge and calculate cost savings associated with hospital days prevented. An overarching theme reflected by Brownell et al. [18] is that "early pharmacist involvement, in coordination with case management colleagues and infectious diseases consult teams, can identify patients poised to receive maximal cost benefit".

In addition to ABSSSI, three studies looked at experiences with other infection types, including that of Brownell et al. introduced above. The experiences of oritavancin in the daily clinical setting in managing Gram-positive osteomyelitis and septic arthritis from native or prosthetic bones and joints are described for a total of 167 patients by Brownell et al. [18] $(n=10)$, Scoble et al. [19] $(n=23)$, and Van Hise et al. [20] $(n=134)$. The paper by Scoble et al. [19] represented a literature review of microbiologically confirmed cases of acute or chronic osteomyelitis. Oritavancin dosing ranged from one dose, for completion of therapy, to ten doses. Maintenance dose (i.e., 800 or $1200 \mathrm{mg}$ ) and dosing frequency (i.e., every 7-14 days) varied between studies and highlights the need to better define these treatment parameters. In an earlier study by Schulz et al. [21], the breakpoint of 7 days was used to differentiate between intravenous oritavancin $1200 \mathrm{mg}$ over $3 \mathrm{~h}$ every dose (i.e., $>7$ days interruption) versus intravenous oritavancin $1200 \mathrm{mg}$ once followed 
by intravenous oritavancin $800 \mathrm{mg}$ over $2 \mathrm{~h}$ (i.e., $\leq 7$ days interruption). In this supplement, Rose and Hudson [22] provide a pharmacokinetic model for a two-dose regimen based on published plasma oritavancin concentrations over time $[14,23]$ that was incorporated into an extended dose treatment algorithm used by Van Hise et al. [20]. These data provide important evidence for the role of oritavancin in the treatment of difficult-to-manage infections. The use of onceweekly options for longer courses of therapy for infections such as osteomyelitis and septic or prosthetic joints could potentially decrease hospital length of stay and readmissions and prevent the need for placement and maintenance of peripherally inserted central catheters or midline catheters, which in turn could decrease central line infections and other complications.

In summary, these real-world studies provide data in excess of 600 patients treated with oritavancin in inpatient and outpatient settings. These practices were incorporated into decision analysis processes and protocols to select appropriate patients for treatment. The study by Van Hise et al. is the largest study focused on osteomyelitis treated with oritavancin and illustrates the consistent application of a system-wide protocol for management of osteomyelitis. This single study exceeds the enrollment reported in a similar recent study by Rappo et al. [24] on the use of dalbavancin for treatment of osteomyelitis.

There are two important caveats to the generalizability of the findings included in this supplement. None of these studies were randomized to standard of care or to a reasonable comparator. Also, much of these data were analyzed retrospectively. However, despite these limitations, clinical and economic outcomes were markedly similar between these studies and fill important gaps in clinical practice. Real-world analyses from large healthcare systems, such as that from Morrisette et al. [25], will provide further insight into the use of long-acting lipoglycopeptides and the clinical judgment of treating physicians. Given the current paucity of treatment options that satisfy the needs of patients, prescribers, and healthcare systems while providing comparable efficacy and safety to traditional antibiotic therapies, these studies are even more valuable.

Patients, providers, payers, and regulators are becoming increasingly aligned in seeking data that clearly address unmet needs and demand evidence to better allow them to evaluate risks and benefits in the clinical setting. RWE has emerged as an important means to understanding the utility of medical interventions in a broader, more representative patient population. Therefore, RWE can give vital insight into treatment effects in more diverse clinical settings and where many patients have multiple comorbidities. RWE can generate hypotheses requiring further investigation in RCTs and also provide answers to some research questions that may be impractical to address through RCTs. Real-world studies can better address novel uses and new interventions by sequentially adapting their design to test evolving questions in the treatment of bacterial infections $[5,26]$.

\section{Compliance with Ethical Standards}

Funding This article was funded by Melinta Therapeutics, Morristown, NJ, USA. This manuscript is part of a supplement wholly funded by Melinta Therapeutics.

Conflict of Interest Dr. Mark Redell has received salary and stock options as an employee of Melinta Therapeutics, manufacturer of oritavancin.

Open Access This article is licensed under a Creative Commons Attribution-NonCommercial 4.0 International License, which permits any non-commercial use, sharing, adaptation, distribution and reproduction in any medium or format, as long as you give appropriate credit to the original author(s) and the source, provide a link to the Creative Commons licence, and indicate if changes were made. The images or other third party material in this article are included in the article's Creative Commons licence, unless indicated otherwise in a credit line to the material. If material is not included in the article's Creative Commons licence and your intended use is not permitted by statutory regulation or exceeds the permitted use, you will need to obtain permission directly from the copyright holder.To view a copy of this licence, visit http://creativecommons.org/licenses/by-nc/4.0/.

\section{References}

1. Klonoff DC, Gutierrez A, Fleming A, et al. Real-world evidence should be used in regulatory decisions about new pharmaceutical and medical device products for diabetes. J Diabetes Sci Technol. 2019. https://doi.org/10.1177/1932296819839996.

2. Sherman RE, Anderson SA, Dal Pan GJ, et al. Real-world evidence-what is it and what can it tell us? New Engl J Med. 2016;375:2293-7.

3. Greenfield S. Making real-world evidence more useful for decision making. Value Health. 2017;20:1023-4.

4. Berger ML, Sox H, Willke RJ, et al. Good practices for real-world data studies of treatment and/or comparative effectiveness: Recommendations from the Joint ISPOR-ISPE Special Task Force on Real-World Evidence in Health Care Decision Making. Value Health. 2017;20:1003-8.

5. FDA. Framework for FDA's real-world evidence program. December 2018. https://www.fda.gov/media/120060/download. Accessed 31 July 2019.

6. US Food and Drug Administration. 21st Century Cures Act. 2018 https://www.fda.gov/regulatoryinformation/lawsenforcedbyfda/ significantamendmentstothefdcact/21 stcenturycuresact/defau 1t.htm. Accessed 28 Aug 2019.

7. Alexander E, Loutit J, Tumbarello M, et al. Carbapenem-resistant Enterobacteriaceae infections: results from a retrospective series and implications for the design of prospective clinical trials. Open Forum Infect Dis. 2017. https://doi.org/10.1093/ofid/ofx063.

8. Moore TJ, Zhang H, Anderson G, Alexander GC. Estimated costs of pivotal trials for novel therapeutic agents approved by the US Food and Drug Administration, 2015-2016. JAMA Intern Med. 2018;178(11):1451-7. 
9. DiMasi JA, Grabowski HG, Hansen RW. Innovation in the pharmaceutical industry: new estimates of R\&D costs. J Health Econ. 2016;47:20-33.

10. Stergiopoulos S, Calvert SB, Brown CA, et al. Cost drivers of a hospital-acquired bacterial pneumonia and ventilator-associated bacterial pneumonia phase 3 clinical trial. Clin Infect Dis. 2018;66(1):72-80.

11. Lee M, Ly H, Möller CC, et al. Innovation in regulatory science is meeting evolution of clinical evidence generation. Clin Pharmacol Ther. 2019;105(4):886-98.

12. Kamath RS, Sudhakar D, Gardner JG, et al. Guidelines vs actual management of skin and soft tissue infections in the emergency department. Open Forum Infect Dis. 2018. https://doi. org/10.1093/ofid/ofx188.

13. Van Hise NW, Tillotson G. Antibiotic development and clinical utilization: a reality check. Ann Microbiol Immunol. 2019;2(1):1011.

14. Melinta. Oritavancin USPI, May 2019.

15. Estrada S, Lodise TP, Tillotson GS, Delaportas D. The real-world economic and clinical management of adult patients with skin and soft tissue infections (SSTIs) with oritavancin: data from two multicenter observational cohort studies. Drugs - Real World Outcomes. 2020. https://doi.org/10.1007/s40801-020-00199-3.

16. Whittaker C, Lodise TP, Nhan E, Reilly J. Expediting discharge in hospitalized, adult patients with skin and soft tissue infections who received empiric vancomycin with oritavancin: description of findings from an institutional pathway. Drugs - Real World Outcomes. 2020. https://doi.org/10.1007/s40801-020-00196-6.

17. Helton B, MacWhinnie A, Minor SB, Lodise TP, Rafferty KD, Allison SL. Early directed oritavancin therapy in the emergency department may lead to hospital avoidance compared to standard treatment for acute bacterial skin and skin structure infections: a real-world retrospective analysis. Drugs - Real World Outcomes. 2020. https://doi.org/10.1007/s40801-020-00201-y.

18. Brownell LE, Adamsick ML, McCreary EK, Vanderloo JP, Ernst EJ, Jackson ER, Schulz LT. Clinical outcomes and economic impact of oritavancin for gram-positive infections: a single academic medical center health system experience. Drugs - Real World Outcomes. 2020. https://doi.org/10.1007/s40801-02000192-w.

19. Scoble PJ, Reilly J, Tillotson GS. Real-world use of oritavancin for the treatment of osteomyelitis. Drugs - Real World Outcomes. 2020. https://doi.org/10.1007/s40801-020-00194-8.

20. Van Hise NW, Chundi V, Didwania V, Anderson M, McKinsey D, Roig I, Sharma A, Petrak RM. Treatment of acute osteomyelitis with once-weekly oritavancin: a two-year, multicenter, retrospective study. Drugs - Real World Outcomes. 2020. https://doi. org/10.1007/s40801-020-00195-7.

21. Schulz LT, Dworkin E, Dela-Pena J, Rose WE. Multiple-dose oritavancin evaluation in a retrospective cohort of patients with complicated infections. Pharmacotherapy. 2018;38(1):152-9.

22. Rose WE, Hutson PR. A two-dose oritavancin regimen using pharmacokinetic estimation analysis. Drugs - Real World Outcomes. 2020. https://doi.org/10.1007/s40801-020-00188-6.

23. Bhavnani SM, Hammel JP, Rubino CM, Moeck G, Jiang H, Bellibas SE, Ambrose PG. Oritavancin pharmacokinetic-pharmacodynamic analyses for efficacy based on data from patients with acute bacterial skin and skin structure infections enrolled in SOLO I and II. Poster A-1309. Presented at: 54th Interscience Conference on Antimicrobial Agents and Chemotherapy (ICAAC), September 5-9, 2014, Washington, DC.

24. Rappo U, Puttagunta A, Shevchenko V, Shevchenko A, Jandourek A, et al. Dalbavancin for the treatment of osteomyelitis in adult patients: a randomized clinical trial of efficacy and safety. Open Forum Infect Dis. 2019. https://doi.org/10.1093/ofid/ofy331.

25. Morrisette T, Miller MA, Montague BT, et al. Long-acting lipoglycopeptides: "lineless antibiotics" for serious infections in persons who use drugs. Open Forum Infect Dis. 2019. https://doi. org/10.1093/ofid/ofz274.

26. Blonde L, Khunti K, Harris SB, et al. Interpretation and impact of real-world clinical data for the practicing clinician. Adv Ther. 2018;35:1763-74. 